\title{
The Fox and the Hedgehog: A Vision of Neuroscience Research in Psychiatry
}

\author{
Honor Hsin ${ }^{1}$
}

Received: 22 December 2014 / Accepted: 23 July 2015 / Published online: 15 August 2015

(C) Academic Psychiatry 2015

The Fox Knows Many Things, but the Hedgehog Knows One Big Thing

(Archilochus, 680-645 B.C.)

In his essay on the writings of Leo Tolstoy, philosopher Isaiah Berlin [1] proposed two types of historical thinkers: those seeking to explain the world through unified principles (hedgehogs) and those seeking to approximate it through various, sometimes disparate, paths of understanding (foxes). As a metaphor for two different approaches to prediction, Berlin's idea raises an intriguing tension between explanation and approximation of natural phenomena. With a new decade of the brain dawning in psychiatry, a similar tension appears to underlie two different ways that neuroscientific thinking encroaches upon clinical understanding and practice: for simplicity, one can be termed a mechanismoriented approach and the other, an outcome-oriented approach. These approaches differ in terms of modeling principles, research methods, implications for drug discovery, and skill set needs (Table 1). The contrast between these two approaches raises interesting considerations for the field of psychiatry on scientific and clinical levels.

\section{Mechanism-Oriented Approach}

Mechanisms in traditional biology are deterministic in nature: part A causes, permits, or inhibits part B in a biological process,

Honor Hsin

honor@stanford.edu

1 Stanford University School of Medicine, Stanford, CA, USA and perturbations in a part will result in predictable changes to the biological state. These parts could be proteins in an intracellular signaling cascade, for example, or cortical nuclei in a neural circuit. Predictions are based on hypothesis testing experiments where the mechanistic model is interrogated by functional changes to specific parts, as in a genetic mutation or drug inhibiting a protein's function, or the surgical ablation of a neuronal population. These experiments generate a unifying model of the biological process in question. The hope here is that therapies can be developed to target a specific biological component ("target-based" drug discovery) for a beneficial clinical effect. In certain subfields of oncology, a cancerous mutation is identified and a pharmacological agent is found that targets this oncogenic component in patients - as in the successful drug imatinib, which inhibits an overactive enzyme found in patients with chronic myelogenous leukemia [2].

This hope is also reflected in recent priorities of the National Institute of Mental Health (NIMH) [3]. Neurobiological models of disease, validated by biological experiments to have high explanatory power, should guide the discovery of targeted therapeutic interventions for various psychiatric disorders. A clinical trial demonstrating the effectiveness of these interventions should, in principle, also show the expected mechanistic effects on neurobiological targets [3]. Pharmaceutical companies have extended this idea to "proof of concept" clinical trials, where new drugs developed through targetbased discovery are tested on small homogeneous populations of target-defined patients [4]. For example, a compound that targets a protein regulated by the Fragile $\mathrm{X}$ gene product can be tested in a subpopulation of patients carrying the same genetic and epigenetic characteristics at the Fragile X gene locus [4]. If the proposed mechanism of Fragile X biology underlying this target choice were valid, these patients would be expected to benefit from the drug. 


\section{Outcome-Oriented Approach}

There may be concerns, however, of such a global reductionist approach. Perhaps neuroscientific research into psychiatric disorders is similar to studying the intestinal microbiome or the earth's climate - a highly dynamic and complex phenomenon rather than a concrete state or process. In these scenarios, it can be difficult to intervene with confidence in such a complex arena. A second approach focused on outcomes, however, may offer an alternative. This approach is probabilistic in nature: whether factor A truly causes factor B cannot be directly ascertained, but accumulated data in large, heterogeneous populations could show a certain probability of association or interaction. Rigorous testing in replication samples will be needed to ensure such a trend holds true and is not merely a statistical fluke. Finally, additional sources of biological information can be synthesized to strengthen the association further. A good example of this approach is found in cardiology: the large-scale Framingham Heart Study showed that high blood cholesterol was a significant risk factor for cardiovascular disease, and studies of patients with familial hypercholesterolemia revealed abnormally elevated rates of cardiovascular events [5]. Although the precise biological mechanism underlying heart disease remains an area of active research, studies have now shown that clinical interventions in lipidemic control can significantly reduce cardiovascular risk [5]. The critical focus here is how to influence clinical outcomes in the most effective manner. The primary mode of clinical intervention is risk reduction rather than mechanisminformed treatment.

In psychiatry, for example, sleep abnormalities have been associated with mood disorders, and there is hope that interventions in circadian biology might mitigate the risk of a mood episode even though the precise mechanistic links between sleep and mood remain under investigation [6]. In another example, genome-wide association studies in large population samples have revealed genetic loci linked to psychiatric disorders such as schizophrenia [7]. Perhaps the functional effects of these genetic changes will reveal a phenotype (e.g., decreased synaptic function in neuronal subtypes) against which new compounds can be screened [8]. This "phenotypic screening" method for drug discovery, which focuses on correcting a biological signature associated with the disease process rather than targeting a specific protein, has been widely used in other fields [9].

\section{Implications}

Whether a gap truly exists between these approaches may remain open to debate; however, there already appears to be a widening gap in the specialization of research training desired of each approach (Table 1). At the investigator level, distinct skill sets appear to be favored. The mechanismoriented researcher will be adept at understanding and applying neurobiological techniques (e.g., molecular biology or brain imaging) for hypothesis-testing experiments and be versed in statistical methods evaluating the results of these small-scale studies. The outcome-oriented researcher will utilize computational modeling and statistical methods for the evaluation of risk factor associations. Advances in machine learning - the design of computational methods that can learn from vast amounts of data - will likely play an important role in future endeavors of this approach. The outcome-oriented researcher will also possess working knowledge of neurobiological techniques that can be adopted on a massive population-level scale. This divergence in training raises the possibility that enhanced exposure to different research methods might help young investigators to reduce tensions between these two approaches and to promote synergy instead.

On a scientific level, potential avenues of synergy include utilizing outcome-oriented methods to define biological risk factors that can be studied mechanistically, in a "local reductionist" paradigm [10]. For example, could the study of sleep processes at a mechanistic level help to identify biological
Table 1 Comparison of two approaches toward the neuroscientific reshaping of clinical psychiatry

\begin{tabular}{lll}
\hline & Mechanism-oriented approach & Outcome-oriented approach \\
\hline $\begin{array}{l}\text { Model } \\
\text { Initial research method }\end{array}$ & $\begin{array}{c}\text { Deterministic } \\
\text { Hypothesis testing in small, } \\
\text { homogeneous populations }\end{array}$ & $\begin{array}{c}\text { Probabilistic } \\
\text { Empirical evaluation and replication } \\
\text { testing in large, heterogeneous } \\
\text { populations }\end{array}$ \\
$\begin{array}{l}\text { Drug discovery } \\
\text { Additional skill set needs } \\
\text { (beyond clinical practice) }\end{array}$ & $\begin{array}{c}\text { Neurget-based } \\
\text { statistical methods }\end{array}$ & $\begin{array}{c}\text { Computational modeling, statistical } \\
\text { methods, machine learning, } \\
\end{array}$ \\
& & scalable neurobiological techniques \\
\end{tabular}

\footnotetext{
${ }^{a}$ Scalability here indicates the degree to which neurobiological techniques can be adapted to large-scale experiments (e.g., genome sequencing is highly scalable at this time due to relative ease and decreasing expense, as compared to functional brain imaging). As technology advances, the number of scalable techniques would be expected to increase
} 
components that, when targeted, may reduce the risk of clinical outcomes such as a manic episode or a suicide attempt? Could a mechanistic understanding of the neural circuitry underlying hedonic behaviors lead to a treatment that reduces the risk of substance use relapse? The NIMH's research domain criteria (RDoC), a system for classifying psychiatric symptoms by neurobiologically derived (and, presumably, evolutionarily conserved) constructs [11], could fit within this role. In other words, RDoC-defined symptoms and their mechanistic underpinnings may be effectively subsumed within an outcome-oriented approach to mental health prognosis and treatment. Another possible path of synergy is the creation of large-scale, probabilistic models of behavior, emotion, and/or cognition by synthesizing and simulating numerous mechanistic sub-processes of the brain - for example, by integrating vast data on neural circuit mechanisms into a comprehensive model for studying the risk of emergent complex properties such as an acute psychotic episode or depressed mood state [12]. The computational powers and techniques required of this task, however, still need significant investment and research at this stage, as reflected in the goals of the Brain Research through the Advancing Innovative Neurotechnologies (BRAIN) initiative [12].

Psychiatry remains one of the most challenging frontiers of human health, and the potential of neuroscience to improve clinical treatment remains great. On a practical level, psychiatrists and trainees will need to be aware of the differences between mechanism-oriented and outcome-oriented approaches, because new therapies discovered by each may serve different clinical niches - a drug target for a homogeneous, genetically defined population, for example, may not be widely applicable to a heterogeneous patient population. Successful interventions discovered through risk reduction models should not be viewed as deterministic for individual patients, however. The application of scientific results to the appropriate clinical context must be appreciated, with the goal of improving mental health for all patients.

Acknowledgments I thank Dr Michael Ostacher for many fruitful discussions on this topic, and Dr Laura Roberts for encouraging this work.

Disclosure The author declares no competing interests.

\section{References}

1. Berlin I. The hedgehog and the fox: an essay on Tolstoy's view of history. 2nd ed. Princeton, NJ: Princeton University Press; 2013.

2. Schiffer C. BCR-ABL tyrosine kinase inhibitors for chronic myelogenous leukemia. New Engl J Med. 2007;357:258-65.

3. Insel T, Gogtay N. National Institute of Mental Health clinical trials: new opportunities, new expectations. JAMA Psychiatry. 2014;71: 745-6.

4. Fishman M. Power of rare diseases: found in translation. Sci Transl Med. 2013;5:201ps11.

5. Libby P. Chapter 241. The pathogenesis, prevention, and treatment of atherosclerosis. In: Longo DL, Fauci AS, Kasper DL, Hauser SL, Jameson J, Loscalzo J, editors. Harrison's principles of internal medicine, 18e. New York, NY: McGraw-Hill; 2012. http://accessmedicine. mhmedical.com.laneproxy.stanford.edu/content.aspx?bookid= 331\&Sectionid=40727018. Accessed December 21, 2014.

6. McClung C. Circadian genes, rhythms and the biology of mood disorders. Pharmacol Ther. 2007;114:222-32.

7. Schizophrenia Working Group of the Psychiatric Genomics Consortium. Biological insights from 108 schizophreniaassociated genetic loci. Nature. 2014;511:421-7.

8. Hyman S. Revolution stalled. Sci Transl Med. 2012;4:155cm11.

9. Swinney D, Anthony J. How were new medicines discovered? Nature Rev Drug Disc. 2011;10:507-19.

10. Kendler K. The structure of psychiatric science. Am J Psychiatry. 2014;171:931-8.

11. Cuthbert B, Insel T. Toward the future of psychiatric diagnosis: the seven pillars of RDoC. BMC Med. 2013;11:126-33.

12. Jorgenson $\mathrm{L}$ et al. The BRAIN initiative: developing technology to catalyse neuroscience discovery. Phil Trans R Soc B. 2015;370: 20140164. 